\title{
LA EDUCACIÓN DE CAMPO EN LAS TESIS SOBRE EDUCACIÓN DE BRASIL: RELEVAMIENTO DE LA PRODUCCIÓN 2013-2014¹
}

\author{
THE RURAL EDUCATION IN THE THESES ABOUT \\ EDUCATION IN BRAZIL: PRODUCTION SURVEY 2013-2014
}

\author{
Maria da Conceição Lopes da Silva Santos \\ ceicelopes@hotmail.com
}

\section{Cecilia Molinari de Rennie}

cecilia.rennie@hotmail.com

\section{RESUMEN}

El propósito de este artículo es identificar espacios para la investigación académica sobre educación de campo en Brasil. Para ello se relevaron las tesis de maestría y doctorado producidas en universidades brasileñas y publicadas en la base BDTD entre 2013 y 2014, encontrando 32 tesis que tratan distintos aspectos de la educación de campo. Estos trabajos fueron sistematizados y clasificados en tres categorías, según el tema, la región geográfica donde se realizó el trabajo de campo y los actores investigados. Se encontró una distribución temática relativamente homogénea en dos temas: políticas de educación rural y pedagogías de educación de campo. Geográficamente, la producción de tesis sobre educación de campo se concentra en la región Sudeste, mientras que en el período 2013-2014 no hay tesis cuyo trabajo de campo se desarrolle en la región Noroeste.

En cuanto a los actores investigados, encontramos una distribución sumamente heterogénea, con un énfasis en la figura del docente, siendo los estudiantes de las escuelas de campo los actores menos investigados. Sumando nuestro aporte a trabajos anteriores, esperamos contribuirá a futuras investigaciones en el marco de los esfuerzos tendientes a promover una educación de campo emancipadora que atienda a las necesidades y expectativas de los hombres y mujeres que habitan las vastas extensiones rurales de Brasil, así como a otras propuestas orientadas a la educación rural en otras regiones de América Latina.

Palabras-clave: educación de campo Brasil; escuela rural; mapeamiento de tesis sobre educación de campo 2013-2014; producción académica de maestría y doctorado.

\footnotetext{
* Licenciada en Geografia (Universidade Estadual de Feira de Santana). Pos-graduada en 2000 (Instituto Pró-Saber), Maestranda en Educación (Universidad de la Empresa, Uruguay). Docente de la Secretaria de Educación del Municipio de Santaluz e del Estado de Bahía, Brasil.

** Doctora en Educación (Universidad de la Empresa); Magister en Educación con énfasis en Investigación en Enseñanza y Aprendizaje (Universidad ORT); Licenciada en Letras (Universidad de la República). Profesora Adjunta de Historia y Filosofía de la Educación (UDELAR); Miembro de la Unidad de Investigación, UDE.

1. Este artículo se enmarca en la línea de investigación "Identidades académicas" desarrollada en el marco del Posgrado en Educación, Facultad de Educación, Universidad de la Empresa (Montevideo-Uruguay), responsable Cecilia Rennie, y en el Proyecto de Tesis Maestría en Educación (Univ. UDE) de Maria da Conceição Lopes da Silva Santos..
} 


\section{ABSTRACT}

This paper aims to identify spaces for academic research on rural education in Brazil. To this end, we reviewed the theses and dissertations from Brazilian universities of 2013-2014 and published in the academic database BDTD, and found 32 theses and dissertations that deal with different aspects of rural education. These were systematized and classified in three categories, according to the topic, the region where the fieldwork was carried out, and the actors that were the subjects of each thesis. We found a relatively homogeneous thematic distribution between policies and pedagogies of rural education. Geographically, the production of theses and dissertations on rural education was concentrated in the South East, whereas in the period 2013-2014 no fieldwork was carried out in the North West in the theses reviewed. Finally, we found a highly heterogeneous distribution in terms of the researched actors, with the great majority of the theses focusing on teachers and very few dealing with students of rural schools.

Adding our contribution to previous works, we hope to contribute to future research aiming at promoting an emancipatory rural education that attends to the needs and expectations of the men and women who inhabit the vast rural areas of Brazil, as well as to other initiatives in the field of rural education in other regions of Latin America.

Keywords: rural education in Brazil; theses mapping; theses on rural education in Brazil 2013-2014; academic production of theses and dissertations

\section{PRESENTACIÓN}

La tasa de analfabetismo en las regiones rurales de Brasil alcanza $23,3 \%$, tres veces mayor que en las áreas urbanas, que es de 7,6\%. Estos datos justifican el interés por parte del gobierno y de otros actores sociales en las distintas dimensiones de la educación de campo, y en el marco de la producción de tesis de posgrado en la última década este interés es especialmente marcado.

Desde la Constitución Federal de 1935 al presente, la educación rural ha sido objeto de numerosas

::98:: políticas por parte del gobierno brasileño. Sin embargo, recién a mediados de los años 80 , con el proceso de redemocratización de Brasil, tiene lugar una conceptualización del "campo" como un espacio propio, que abriga poblaciones heterogéneas con necesidades específicas. A partir de este momento, y en el contexto de movimientos sociales orientados a la reapropiación de las tierras por los trabajadores rurales (en particular el Movimiento Sin Tierra - MST), se desarrollan numerosas iniciativas orientadas a lo que ahora se denominará educación de campo. Según el IBGE (Instituto Brasileiro de Geografía e Estatística), se consideran parte del área rural aquellos domicilios situados fuera del perímetro urbano de un municipio (Brasil, 2010: en línea). Para definir "perímetro urbano" debemos recurrir al Decreto-Ley 311, de 1938, donde se establece que las ciudades (sedes de municipios) y las villas (sedes de distritos) son urbanas mientras que el territorio restante es rural, correspondiendo a las prefecturas realizar esa definición a través de leyes. Más allá de esta conceptualización jurídica, hay otras formas de concebir lo urbano y lo rural: si analizamos la producción económica en ambas regiones, la ciudad ocupa un espacio específico bien distinto del espacio rural, y esta distinción depende del modo de producción (Lefebvre, 2008): es responsabilidad del campo el sector primario, mientras que en las ciudades tienen lugar las actividades de los sectores secundario y terciario, que desarrollan en exclusividad. Sin embargo, cada vez es más evidente que esta delimitación no sucede de modo rígido, ya que algunas áreas rurales del país poseen la misma estructura (agua corriente, luz eléctrica, sistema de telefonía e industrialización) que los grandes centros urbanos. En el caso de Brasil, es complejo definir el campo como un territorio técnicamente delimitado, ya que la cercanía del municipio no es el mejor criterio para esta distinción y en los hechos nos encontramos en un terreno de excepciones, donde los sectores de la economía y las características histórico-culturales atraviesan esos espacios y sus habitantes; la diversidad lingüística, étnica y cultural de los habitantes "del campo" complica aún más agrupar estos sujetos bajo la misma etiqueta.

En el marco de las iniciativas gubernamentales y sociales a favor de un acceso igualitario de todos los ciudadanos a una educación emancipadora, se vienen desarrollando una serie de iniciativas para mejorar la escuela de campo en todas sus dimensiones; nuestro trabajo pretende ser un aporte a estos esfuerzos, sobre todo a nivel de trabajos de posgrado, planteando el panorama actual y sugiriendo algunos caminos para futuras investigaciones. 


\section{CONTEXTO SOCIO-HISTÓRICO: LAS POLÍTICAS DE EDUCACIÓN DE CAMPO EN BRASIL}

Si bien a partir de la Constitución Federal de 1935 Brasil ha desarrollado sostenidamente políticas dedicadas a la educación de campo, es posible agrupar estos esfuerzos en cuatro etapas: 1935 -1963, 1964-1984, 1985-2001, y de 2002 al presente, correspondiendo a distintos momentos en la historia de Brasil (Tabla 1).

Tabla 1 - Políticas de educación rural en Brasil, 1935-1963

\begin{tabular}{|l|l|}
\hline Período & Acontecimientos \\
\hline $1935-1963$ & $\begin{array}{l}\text { De las primeras políticas públicas sobre educación } \\
\text { de campo al inicio del gobierno militar. }\end{array}$ \\
\hline $1964-1984$ & Período de dictadura militar \\
\hline $1985-2000$ & Período de redemocratización del Brasil \\
\hline 2000 - presente & Gobierno del Partido de los Trabajadores \\
\hline
\end{tabular}

Fuente: Autora.

\subsection{Período 1935-1963}

A partir de la primera Constitución Federal de Brasil comienzan a desarrollarse políticas educativas orientadas al área rural del país. La Constitución de 1935 determina que el $20 \%$ del presupuesto anual de educación estará destinado a la educación rural (actualmente existe en el país el Pronacampo - un programa que estructura físicamente las escuelas, invierte en transporte y formación de los profesionales, además del Programa Dinero Directo a la Escuela o PDDE que asigna cerca de $R \$ 12$ mil por años a las escuelas con hasta 50 alumnos). Esta cifra puede parecer irrisoria para el lector contemporáneo, pero considerada en su contexto histórico representa un importante reconocimiento de las necesidades de educación de esta franja de la población. Las iniciativas durante este período reflejan un doble interés: por una parte, continuar ampliando la alfabetización de la población rural iniciada durante la época de la Colonia; en este sentido cabe destacar la creación por Ley Federal en 1945 de centros de entrenamiento de profesores, y la obligación, impuesta por la Constitución Federal de 1946 de que las empresas (tanto urbanas como agrícolas) con más de 100 empleados proporcionen enseñanza primaria gratuita tanto para los empleados como para sus hijos. Por otra parte, encontramos una serie de medidas orientadas a evitar las migraciones masivas a las zonas industrializadas, valorizando la cultura rural. La Tabla 2 sintetiza las principales iniciativas de este período.

Tabla 2 - Políticas de educación rural en Brasil, 1935-1963

\begin{tabular}{|l|l|l|}
\hline Año & Acto & Resoluciones \\
\hline 1935 & Constitución Federal & $\begin{array}{l}\text { Art.121: atender la educación rural para afincar } \\
\text { al hombre de campo } \\
\text { Art.156: 20\% del presupuesto anual de } \\
\text { educación para enseñanza rural }\end{array}$ \\
\hline 1937 & $\begin{array}{l}\text { Constitución Federal: } \\
\text { Creación de la Sociedad Brasileña } \\
\text { de Educación Rural }\end{array}$ & $\begin{array}{l}\text { Promoción de enseñanza de arte y folklore } \\
\text { rurales. } \\
\text { Educación como "canal de difusión ideológica". }\end{array}$ \\
\hline 1945 & $\begin{array}{l}\text { Ley Federal: } \\
\text { Creación de la Comisión Brasileña } \\
\text { de Educación de las Poblaciones } \\
\text { Rurales --CBAR }\end{array}$ & $\begin{array}{l}\text { Proyectos educativos en zona rural } \\
\text { Centros de entrenamiento para profesores. } \\
\text { Clubes agrícolas y Consejos Comunitarios } \\
\text { Rurales. }\end{array}$ \\
\hline
\end{tabular}




\begin{tabular}{|c|c|c|}
\hline 1946 & Constitución Federal & $\begin{array}{l}\text { Art. 168:III Empresas urbanas y agrícolas con } \\
\text { más de } 100 \text { empleados deben proporcionar } \\
\text { enseñanza primaria gratuita para los } \\
\text { empleados y sus hijos. }\end{array}$ \\
\hline & $\begin{array}{l}\text { Decreto-Ley } 9613 \\
\text { Ley Orgánica de Enseñanza } \\
\text { Agrícola }\end{array}$ & $\begin{array}{l}\text { Creación de Misiones Rurales para promover } \\
\text { alfabetización }\end{array}$ \\
\hline 1949 & $\begin{array}{l}\text { Seminario: } \\
\text { Creación de Misiones Rurales }\end{array}$ & $\begin{array}{l}\text { preparación de líderes y agentes comunitarios } \\
\text { para alfabetización }\end{array}$ \\
\hline 1961 & $\begin{array}{l}\text { Ley de Directrices y Bases de la } \\
\text { Educación (LDBE) }\end{array}$ & $\begin{array}{l}\text { Art. 105: incremento de escuelas en zona rural. } \\
\text { Favorecer adaptación al medio y estímulo de } \\
\text { vocaciones profesionales }\end{array}$ \\
\hline
\end{tabular}

Fuente: Autora.

Durante este período, emergen algunas figuras pioneras en el campo de la Educación que abrieron el camino para que décadas más tarde otros pensadores trabajaran en una profunda reconceptualización de la educación rural desde una perspectiva crítica. En la década del 20 ingresa en la vida política el bahiano Anísio Teixeira, bajo cuya influencia se organizará por primera vez un sistema educativo en Brasil. Teixeira fue Inspector General de Enseñanza de Bahía y posteriormente Director de Instrucción Pública de Enseñanza de este mismo estado, para luego asumir la Secretaría de Educación y Cultura del Distrito Federal, participando del Manifiesto de los Pioneros de la Escuela Nueva (1932) y la Asociación Brasileña de Educación (ABE); fue también uno de los creadores de la Universidad del Distrito Federal (UDF). Afrânio Peixoto, contemporáneo de Teixeira, también nacido en el interior de Bahía, fue otro intelectual que percibió claramente el rol de la escuela -especialmente la enseñanza secundaria-- como reproductora de las desigualdades sociales."

Sobre fines de este período emerge también la gran figura brasileña en el campo de la educación, Paulo Freire. Freire será coordinador del Programa Nacional de Alfabetización en 1964, extendiendo

::100:: el alcance del escolanovismo, sobre todo en cuanto a la alfabetización de adultos. El contexto sociopolítico de Brasil en los años de dictadura no permitió que las ideas de Freire se volcaran en las políticas de alfabetización, pero su influencia en el campo de la educación es incalculable, no sólo en Brasil sino fuera de este país donde su pensamiento dio lugar a la corriente de pedagogía crítica en los años 70-80.

\subsection{La educación durante la dictadura: 1964- 1983}

Tabla 3 - Políticas de educación de campo durante la dictadura militar

\begin{tabular}{|c|c|c|}
\hline Año & Acto & Resoluciones \\
\hline 1967 & $\begin{array}{l}\text { Ley 5.379: } \\
\text { MOBRAL }\end{array}$ & Creación del Movimiento Brasileño de Alfabetización \\
\hline 1971 & LDBE n $5692 / 71$ & $\begin{array}{l}\text { Currículo orientado a atención de peculiaridades locales } \\
\text { Formación de profesores adecuada a diferencias } \\
\text { culturales } \\
\text { Acceso del hombre de campo a la educación rural } \\
\text { Responsabilidad de las empresas en formación de } \\
\text { empleados }\end{array}$ \\
\hline 1971 & $\begin{array}{l}\text { I Plan Nacional de } \\
\text { Desarrollo (PND) }\end{array}$ & $\begin{array}{l}\text { Valorización de la escuela rural } \\
\text { Oportunidades de manifestación cultural del hombre de } \\
\text { campo } \\
\text { Extensión de acceso } \\
\text { Homogeneización del calendario escolar para toda la } \\
\text { escuela rural } \\
\text { Unidad escolar rural como agente de cambios y } \\
\text { transformaciones sociales }\end{array}$ \\
\hline
\end{tabular}


La dictadura militar, que se extendió entre 1964 y 1985, cambia radicalmente el escenario, propiciando la implantación de políticas de orientación tecnicista inspiradas en los principios de racionalidad, eficiencia y productividad. En 1967 con la implementación del MOBRAL ${ }^{2}$ (Movimento Brasileiro de Educação) tiene lugar un descenso en los índices de analfabetismo del país ya que logra alcanzar la casi totalidad de los habitantes de Brasil, principalmente en la zona rural. Este éxito, sin embargo, fue duramente cuestionado debido al énfasis en la alfabetización y el descuido de la literalidad que caracterizaron el MOBRAL.

Hacia fines de la dictadura militar, según datos de INEP (BRASIL, 2007) el diagnóstico de la educación de campo señalaba como principales problemas los siguientes:

Insuficiencia y precariedad de las instalaciones físicas de la mayoría de las escuelas;

Dificultades de acceso de los profesores y alumnos a las escuelas, en razón de carecer de un sistema adecuado de transporte escolar;

Falta de profesores habilitados y con efectividad, lo cual provoca una constante rotación

Currículo escolar que privilegia una visión urbana de la educación y el desarrollo

Ausencia de asistencia pedagógica y supervisión escolar en las escuelas rurales

Predominio de clases multiseriadas con educación de baja calidad

Falta de actualización de las propuestas pedagógicas de las escuelas rurales

Bajo desempeño escolar de los alumnos y elevadas tasas de distorsión edad-serie

Bajos salarios y sobrecarga de trabajo de los profesores, en comparación con los que actúan en la zona urbana.

En 1977 el índice de analfabetismo en la población rural de Brasil era de 29,1\%; cinco años más tarde no había reducción sino aumento en esta cifra - que pasó a ser de 29,9 \%-- lo cual muestra la ineficacia del MOBRAL en este contexto. Al promover un análisis comparativo de la retención escolar en las áreas rurales y urbanas se evidencia que en las ciudades ésta fue mucho más eficiente, posiblemente en función de la falta de estructura rural, como muestra la Tabla 4.

Tabla 4 - Analfabetismo en Brasil en la década de los 80

\begin{tabular}{|c|c|c|c|}
\hline $\begin{array}{c}\text { Edad de } \\
\text { escolarización } \\
\text { obligatoria }\end{array}$ & Total Brasil & Área urbana & Área rural \\
\hline De 5 a 14 anos & $\begin{array}{c}42,1 \% \text { no } \\
\text { frecuentaban la } \\
\text { escuela }\end{array}$ & $\begin{array}{c}29,3 \% \text { de este } \\
\text { contexto estaban en } \\
\text { las ciudades }\end{array}$ & $\begin{array}{c}63,5 \% \text { de estos niños } \\
\text { viven en el campo }\end{array}$ \\
\hline
\end{tabular}

Fuente: Autora.

\subsection{La educación de campo en la redemocratización de Brasil: 1985- 2001}

El proceso de redemocratización del país, que comienza en 1985, propicia una serie de manifestaciones populares, sindicales y de intelectuales en torno al proceso que generará la nueva Constitución, caracterizado por el deseo de democratización del país, la afirmación de una cultura de derechos y un espacio de participación popular en las políticas públicas.

En este marco se desarrolló el proyecto de regulación de las legislación educativa que culminó con la nueva Ley de Directrices y Bases de la Educación (LDB, Ley N 9.394, de diciembre de 1996), que a su vez desencadenó un conjunto de normativas para regular el funcionamiento y la organización administrativa y curricular de las escuelas en Brasil, incluyendo nuevos Planes de 
Educación; Directrices Curriculares Nacionales; Directrices Operacionales Nacionales, Parámetros Curriculares Nacionales y Fondo de Desarrollo y Mantenimiento del Magisterio.

Este proceso estuvo marcado por la descentralización administrativa y presupuestaria y por la discusión y movilización para la elaboración de las Directrices Operacionales para la Educación Básica en las Escuelas de Campo. La siguiente tabla sintetiza los acontecimientos más significativos para la educación de campo entre 1980 y 2001.

Tabla 5 - Políticas de educación de campo 1980-2001

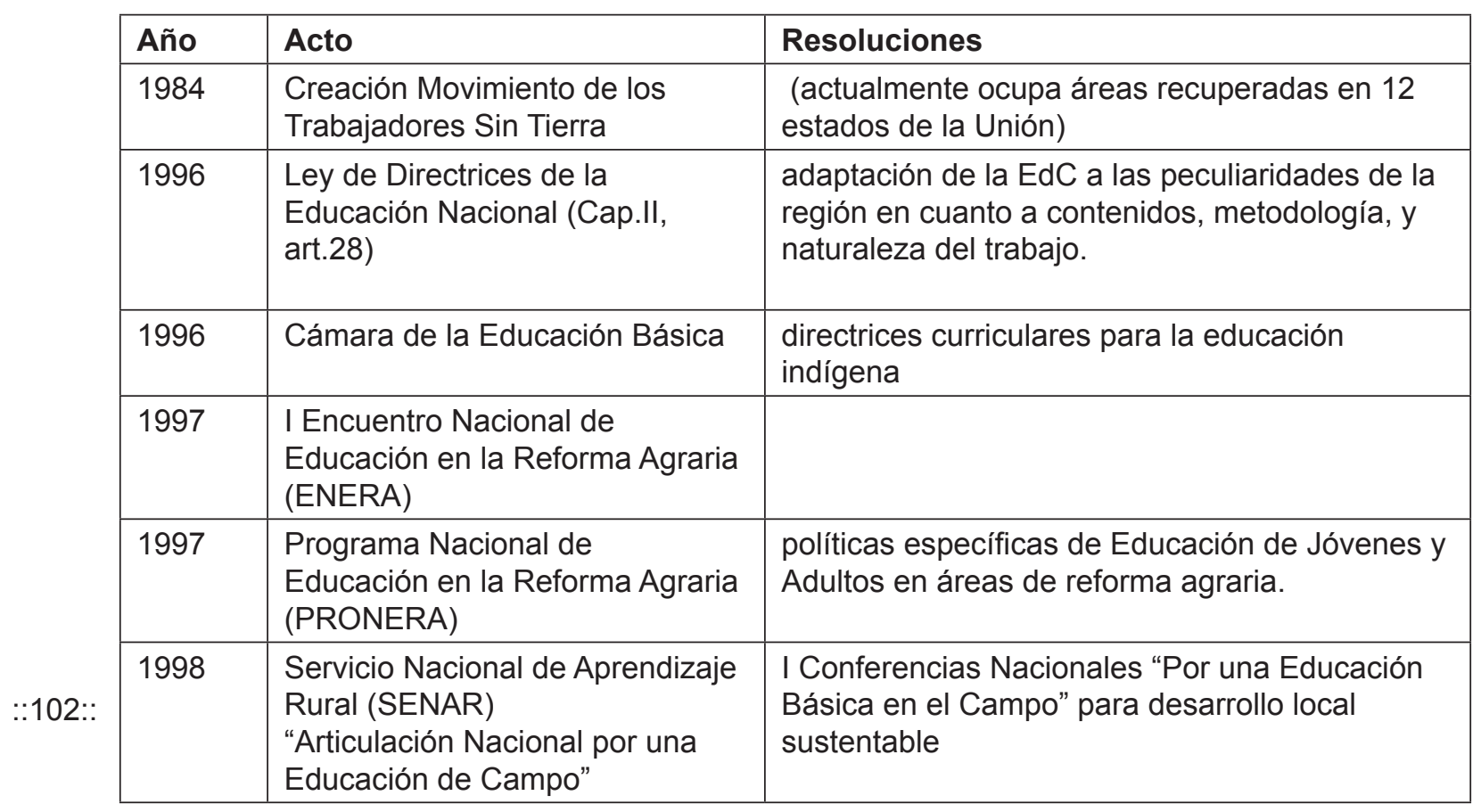

Fuente: Autora.

A partir de las medidas mencionadas, y con la municipalización de la enseñanza fundamental, las sugerencias, decisiones legales y acción de las nuevas entidades comienzan a ser efectivizadas en el campo (Frigotto, 2010:23).

Sin embargo, la aplicación de estas políticas no resolvió la cuestión que ya planteaban las primeras iniciativas de educación rural: la necesidad de emigrar para continuar sus estudios y eventualmente para acceder a puestos de trabajo acordes a la preparación académica de estos sujetos. Esta preocupación marcó la agenda para la siguiente etapa en la evolución de la educación de campo en Brasil, en la cual trabajaron diversos actores incluyendo movimientos sindicales, organizaciones sociales, Universidades, ONG, Secretarías estatales y municipales de educación y otros órganos de gestión pública para implementar las normas y leyes ya existentes sobre educación de campo.

\subsection{La educación de campo durante el gobierno del Partido de los Trabajadores: 2002 al presente}

A comienzos de 2001, el Consejo de Educación, a través de la Cámara de Educación Básica, elaboró las Directrices Curriculares Nacionales para la Educación Infantil, Enseñanza fundamental y Media, para la Educación de Jóvenes y Adultos, la Educación Indígena y la Educación Especial, la Educación Profesional y la Formación Docente a nivel medio en la modalidad normal, además de realizar audiencias públicas para escuchar las propuestas de los representantes de los órganos normativos estatales y municipales, de los movimientos sociales de campo, las Universidades y ONGs para la implementación de las Directrices Operacionales para la Educación Básica en las 
Escuela de Campo que fue homologada por el ministerio de Educación el 12 de marzo de 2002. Las directrices reconocen el modo de vida social propio del campo, no circunscripta a un espacio geográfico sino vinculada a los pueblos que habitan en las zonas rurales o los que viven en las ciudades de los 4.485 municipios rurales de Brasil.

Tabla 6 - Políticas públicas a partir de 2002

\begin{tabular}{|l|l|l|}
\hline Año & Acto & Resoluciones \\
\hline 2004 & $\begin{array}{l}\text { Programa Brasil } \\
\text { Alfabetizado - Ley } n^{\circ} \\
10.880 / 2004\end{array}$ & $\begin{array}{l}\text { Ampliación oferta de grupos } \\
\text { subvención de materiales escolares y gastos de } \\
\text { alimentación y transporte para alfabetizadores y } \\
\text { estudiantes }\end{array}$ \\
\hline 2004 & $\begin{array}{l}\text { Organizaciones sindicales } \\
\text { e gubernamentales por una } \\
\text { educación en campo }\end{array}$ & $\begin{array}{l}\text { II Conferencias Nacionales "Por una Educación Básica en } \\
\text { el Campo" para desarrollo local sustentable }\end{array}$ \\
\hline 2013 & $\begin{array}{l}\text { Ley } n^{\circ} 12.695 / 2012 \\
2013: \text { Programa Nacional } \\
\text { de Educação do Campo } \\
\text { (Pronacampo), }\end{array}$ & $\begin{array}{l}\text { Computa en el FUNDEB las matrículas de estudiantes de } \\
\text { campo ofrecidas por instituciones comunitarias, sin fines } \\
\text { de lucro, actuando en convenios con el poder público en } \\
\text { la formación alternativa. }\end{array}$ \\
\hline 2013 & $\begin{array}{l}\text { Decreto no 1.062 - 2013: } \\
\text { Programa Nacional dos } \\
\text { TerritóriosEtnoeducacion de la infraestructura edilicia } \\
\text { Formación inicial y continuada de profesores } \\
\text { Facilitación de materiales didácticos }\end{array}$ & $\begin{array}{l}\text { Apoyo técnico y financiero del MEC a los sistemas de } \\
\text { enseñanza escolar indígena }\end{array}$ \\
\hline
\end{tabular}

Fuente: Autora.

Las políticas implementadas en este período, sintetizadas en la Tabla 6, marcan un giro en la forma en que se enfoca la educación de campo, a partir de un Proyecto Político Pedagógico vinculado los desafíos, las aspiraciones, la historia y la cultura de la población de campo. Esta nueva mirada "projeta o campo como espaço de democratização da sociedade brasileira e de inclusão social, e que projeta seus sujeitos como sujeitos de história e de direitos; como sujeitos coletivos de sua formação enquanto sujeitos sociais, culturais, éticos, políticos." (Arroyo, 2008: 152)

\section{METODOLOGÍA}

La necesidad de una revisión de la literatura se justifica por el volumen de información accesible y por la necesidad de sistematizar la información relevante de modo que pueda ser asimilada por el investigador (Cooper, 1985; Cooper \& Hedges, 2009; Randolph, 2009, entre otros). Los distintos tipos de revisión de la literatura suelen clasificarse utilizando la taxonomía de Cooper (1985), quien identifica cinco características: Foco, Objetivo, Perspectiva, Alcance, Organización y Audiencia. El Foco de una revisión se refiere al material que constituye el centro de interés del autor; nuestro Foco en este caso es "Prácticas y aplicaciones", ya que nos interesa recoger tesis y disertaciones. El Objetivo es lo que se quiere lograr con la revisión; en este caso, identificar los temas centrales en tesis recientes sobre educación de campo en Brasil así como las cuestiones menos investigadas. En cuanto a la Perspectiva, adoptamos en este trabajo una perspectiva "neutra", en el sentido que se trata de un trabajo descriptivo, donde no se lleva a cabo una evaluación crítica de las tesis relevadas. En relación con el Alcance del trabajo, esta revisión es un relevamiento exhaustivo de todas las tesis sobre educación de campo en Brasil publicadas en un período de dos años (2013 y 2014) en la base de datos BDTD (Biblioteca Digital Brasileira de Teses e Dissertações). Con respecto a la Organización, al estar trabajando con un corpus relativamente pequeño (32 tesis) hemos organizado estos datos con un criterio conceptual -identificando los temas centrales de las 
tesis relevadas-- y con un criterio metodológico - agrupando los trabajos según los métodos de investigación utilizados. La Audiencia a la cual apuntamos son principalmente investigadores en el área de la educación rural.

Tabla 7 - Tipo de revisión

\begin{tabular}{|l|l|l|}
\hline \multicolumn{2}{|c|}{ Tipo de revisión, según la clasificación de Cooper $(1985,1988)$} \\
\hline Característica & \multicolumn{1}{|c|}{ Tipo } & \multicolumn{1}{c|}{ Descripción } \\
\hline Foco & Prácticas o aplicaciones & $\begin{array}{l}\text { Tesis y disertaciones sobre } \\
\text { educación de campo }\end{array}$ \\
\hline Objetivo & $\begin{array}{l}\text { Identificación de temas } \\
\text { centrales }\end{array}$ & $\begin{array}{l}\text { Aspectos de la educación de campo } \\
\text { investigados }\end{array}$ \\
\hline Perspectiva & Representación neutral & Exposición descriptiva \\
\hline Alcance & Exhaustivo & $\begin{array}{l}\text { Totalidad de tesis sobre educación } \\
\text { de campo publicadas en 2013 y } \\
\text { 2014 en la BDTD }\end{array}$ \\
\hline Organización & Conceptual & $\begin{array}{l}\text { Identificación y clasificación de focos } \\
\text { de interés }\end{array}$ \\
\hline Audiencia & Investigadores en general & $\begin{array}{l}\text { Investigadores en educación; } \\
\text { tesistas }\end{array}$ \\
\hline
\end{tabular}

Fuente: Adaptado de Cooper, 1985 y 1988.

En cuanto al desarrollo de la revisión, seguimos las etapas usuales en este tipo de trabajo (Cooper 1985; 1988; Cooper \& Harris, 2009; Randolph, 2009, entre otros): formulación del problema, recolección de datos, evaluación y sistematización de datos; análisis e interpretación, y presentación

$:: 104::$ de los resultados (Randolph, 2009: 4 y ss).

\subsection{Formulación del problema y delimitación de criterios de inclusión/exclusión}

Siguiendo estas etapas, comenzamos planteando la pregunta central que pretendemos responder con el relevamiento: ¿Qué aspectos de la educación de campo constituyen el foco de las tesis y disertaciones producidas recientemente en Brasil y publicadas en el repositorio de la BDTD? Con esta pregunta pretendemos emprender una exploración de este campo de conocimiento que funcionará como plataforma para nuestros respectivos proyectos de investigación. Como criterios de inclusión, los trabajos relevados deberían haber sido producidos en universidades del Brasil, y estar escritos en portugués. Dado que en la etapa de relevamiento de antecedentes de esta investigación encontramos trabajos que se ocupan de las tesis y disertaciones producidas en años anteriores, tomamos criterio de exclusión que la tesis o disertación no hubiera sido publicada fuera del período 2013- 2014.

\subsection{Recolección de datos}

La segunda etapa, recolección de los datos, fue realizada en Internet, en la base de datos de la BDTB. Comenzamos con el término de búsqueda más amplio, "MST", dado que los proyectos de educación de campo actuales están, como se desprende de la descripción del contexto sociohistórico, vinculados al Movimiento Sin Tierra. Esta búsqueda arrojó 66 resultados. De éstos, 6 corresponden a tesis sobre educación de campo publicadas entre 2013 y 2014 . Luego realizamos una nueva búsqueda utilizando la expresión "educação do campo", obteniendo 13 resultados correspondientes a tesis publicadas entre 2013 y 2014. A continuación, utilizamos como término de búsqueda "escola do campo", obteniendo 11 trabajos cumplen con los criterios de publicación entre 2013 y 2014, incluyendo 3 que ya habían sido relevadas en búsquedas anteriores, con lo cual recogimos 8 tesis. Por último, realizamos una búsqueda con los términos "escola rural", obteniendo 9 resultados, 
de los cuales 5 trabajos ya habían sido relevados en las búsquedas anteriores, obteniendo así 4 resultados. En este momento, consideramos haber alcanzado el punto de saturación.

En total, se relevaron 32 tesis de maestría y doctorado sobre temas relativos a la educación rural en Brasil publicados entre enero de 2013 y diciembre de 2014 en la base de datos BDTD. La tabla siguiente muestra sinópticamente estos resultados:

Tabla 8 - Relevamiento realizado en la base de datos BDTD

\begin{tabular}{|l|r|r|r|}
\hline Términos de búsqueda & Resultados & Repetidos & Relevados \\
\hline "MST" & 6 & 0 & 6 \\
\hline "educação do campo" & 13 & 0 & 13 \\
\hline "escola do campo" & 11 & 3 & 8 \\
\hline "educação rural" & 9 & 5 & 4 \\
\hline TOTAL TESIS RELEVADAS & & & 32 \\
\hline
\end{tabular}

Fuente: Autora.

En total, relevamos 32 tesis que cumplían con los criterios que habíamos establecido para la búsqueda.

\subsection{Evaluación de los datos}

En la tercera etapa de este trabajo, procedimos a sistematizar los trabajos relevados, tabulándolos con ayuda de una planilla de Microsoft Excel ${ }^{\mathrm{T}}$, donde registramos fechas y criterios de búsqueda, datos bibliográficos de los trabajos, datos de las universidades y regiones donde se había realizado el trabajo de campo, información sobre la investigación (palabras clave, objetivo, población investigada, instrumentos metodológicos, hallazgos), y notas de lectura (aspectos de la educación de campo tratados y otros comentarios).

En base a esta tabla, realizamos una clasificación temática, identificando las áreas que habían sido objeto de investigación en las tesis de la base de datos de la BTDB, una clasificación geográfica, según las regiones del Brasil donde se habían llevado a cabo estas investigaciones, y una clasificación metodológica, según qué sujetos habían sido investigados en estos trabajos. En la siguiente sección exponemos los resultados obtenidos.

\section{RESULTADOS}

\section{1. Áreas temáticas}

Las tesis relevadas pueden agruparse en dos grandes áreas temáticas, que hemos denominado Pedagogía y Políticas, con 16 tesis en cada categoría.

A la dimensión Pedagogía corresponden tesis cuyo centro de interés temático son cuestiones relativas a la enseñanza en las escuelas de campo: cómo se enseña, qué se enseña, quién enseña en estas escuelas. Dentro de esta dimensión se destaca un tema central, la formación docente, que es el centro de interés de 12 de las 16 tesis relevadas. 


\section{Gráfico 1: Área temática "Pedagogía”}

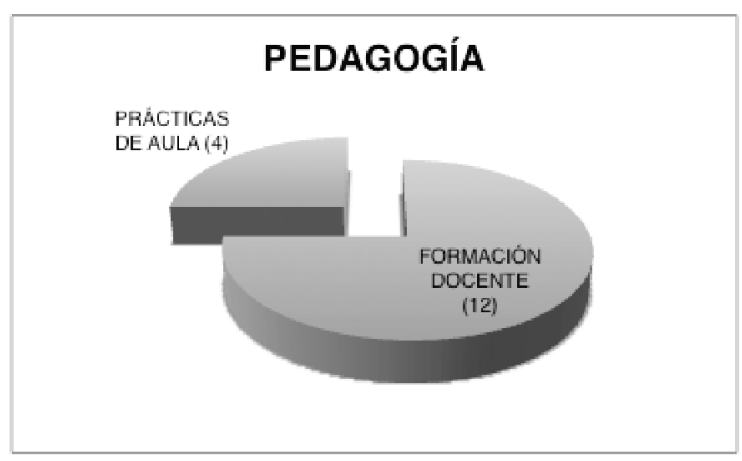

Fuente: Autora.

De las tesis relevadas, Aquino (2013), Freitas (2013) y Pinto (2013) tratan cuestiones relativas a las competencias de los educadores en formación en distintas Licenciaturas en Educación de Campo; Barradas (2013), Lopes (2013) y Sperandio (2013) se centran en la formación continuada y profesionalización de los docentes de campo, mientras que Ferreira (2014), Santi (2013) y Weiss (2013) se ocupan de relatos de docentes de campo en distintos momentos de su carrera profesional. Los restantes trabajos sobre formación docente (Lopes, 2013; Meneses, 2013; Moreira, 2013) tratan otros aspectos como representaciones sobre "educación de campo" y la relación entre formación docente y áreas temáticas o programas específicos.

Los 4 trabajos restantes tratan sobre distintos temas relativos a la enseñanza y aprendizaje, en un caso la enseñanza de lengua portuguesa (dos Santos, 2013), educación ambiental (de Campos 2014), prácticas de literacidad (dos Santos, 2013), y cultura corporal (Lavoura, 2013).

Esta distribución es consistente con los datos relevados en 2006 por Araújo (2007), quien encuentra

::106:: que la mayoría de las tesis publicadas en los 6 años anteriores (2002- 2006) trataba sobre la formación de los profesores y maestros de campo, mostrando una continuidad en esta tendencia. Por otra parte, este énfasis dice de una creencia presente en la sociedad brasileña de que no puede haber cambio educativo sin docentes preparados para llevarlo adelante, lo cual ubica al docente en el centro de atención a expensas de otros factores.

La categoría Políticas reúne las restantes 16 tesis relevadas, incluyendo trabajos que exploran la elaboración e implementación de los distintos programas, políticas y normativas que regulan la educación de campo en Brasil. Dentro de este grupo encontramos una distribución algo más homogénea, identificando cuatro temas: diseño, implementación, impacto e historia de las políticas de educación de campo en Brasil.

\section{Gráfico 2 - Área temática "Políticas"}

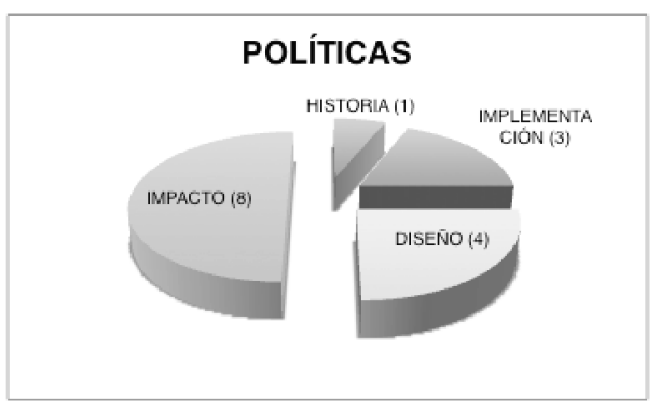

Fuente: Autora.

Hemos clasificado como pertenecientes a la dimensión Impacto de la educación de campo aquellos trabajos que tratan de cómo afectan a los individuos y a las comunidades los programas de educación de campo, encontrando una distribución homogénea en 2 ejes temáticos, según se trate del impacto de la educación de campo sobre las identidades individuales o comunitarias. Cuatro tesis tratan 
sobre el impacto de los programas en los proyectos a futuro de los beneficiarios, particularmente en relación con su inserción laboral y posibilidades de continuar estudiando (Moreira 2013, Kuhn 2013; de Araújo 2013; Gimenes, 2014). Las 4 restantes se centran en la educación de campo como formadora de identidades de los distintos actores (Larchet 2013; Lavoura 2013; Oliveira 2014; Pereira, 2013).

En cuanto a los otros subgrupos dentro de la categoría Políticas, 5 tesis (Basso, 2013; de Aguilar, 2013; Menin da Silva, 2013; Priebe, 2013; Tondin, 2013) tratan sobre el diseño de las mismas, enfocándose particularmente en la adecuación de las mismas a las necesidades específicas de sus beneficiarios. Además, 3 tesis (Ferolla, 2013; Jesus, 2013; Paula, 2013) se ocupan de cuestiones relativas a la implementación de las políticas sobre educación de campo en contextos particulares, enfatizando las dificultades que encuentran los distintos actores. El trabajo restante (Pereira, 2013) es el único que trata sobre los antecedentes de la educación de campo, enfocándose en las políticas y normativa gubernamentales entre 1946 y 1961.

Hasta 2006, las políticas de educación de campo eran una de las áreas temáticas con menor producción a nivel de tesis de posgrado (Araújo, 2007; en este sentido podemos ver un creciente interés a medida que se multiplican las iniciativas en el marco del proyecto educativo de los gobiernos socialistas de Brasil a partir de 2002. Más aún, dentro de este tema vemos que el énfasis cambió de la historia de las políticas educativas implementadas en el marco del MST, que Araújo (2007) identifica como uno de los temas de mayor producción en ese momento al estudio de las políticas actuales implementadas en los últimos años.

\section{Gráfico 3 - Temas en las Tesis sobre Educación de Campo, 2013-2014}

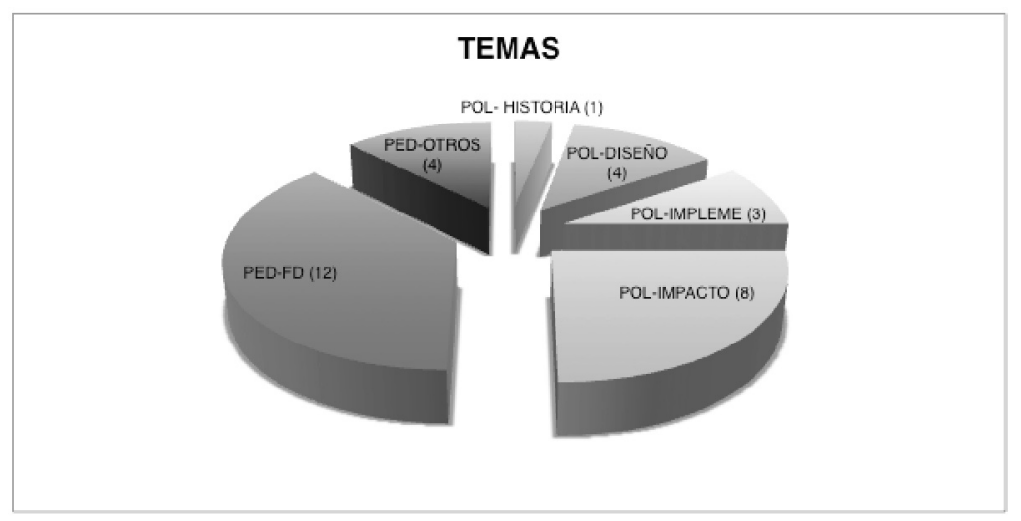

Fuente: Autora.

\subsection{Regiones donde se realizó el trabajo de campo}

La división regional de Brasil por el IBGE (BRASIL, 2010), identifica cinco regiones geográficas: Norte (estados de Acre, Amapá, Amazonas, Pará, Rondonia, Roraima y Tocantins), Nordeste (Alagoas, Bahía, Ceará, Maranhao, Paraíba, Pernambuco, Piauí, Rio Grande del Norte y Sergipe), CentroOeste (Goiás, Mato Grosso, Mato Grosso del Sur y el Distrito Federal), Sudeste (Espírito Santo, Minas Gerais, Río de Janeiro y Sao Paulo), y Sur (Paraná, Rio Grande do Sul y Santa Catarina).

En cuanto a las regiones donde se realizaron las investigaciones relevadas, encontramos que 24 tesis se ocupan de la zona Sudeste, 4 del Sur, 2 del Nordeste y ninguna (0) investiga la educación rural en la zona Centro-Oeste. 


\section{Gráfico 4 - Regiones donde se realizó el trabajo de campo}

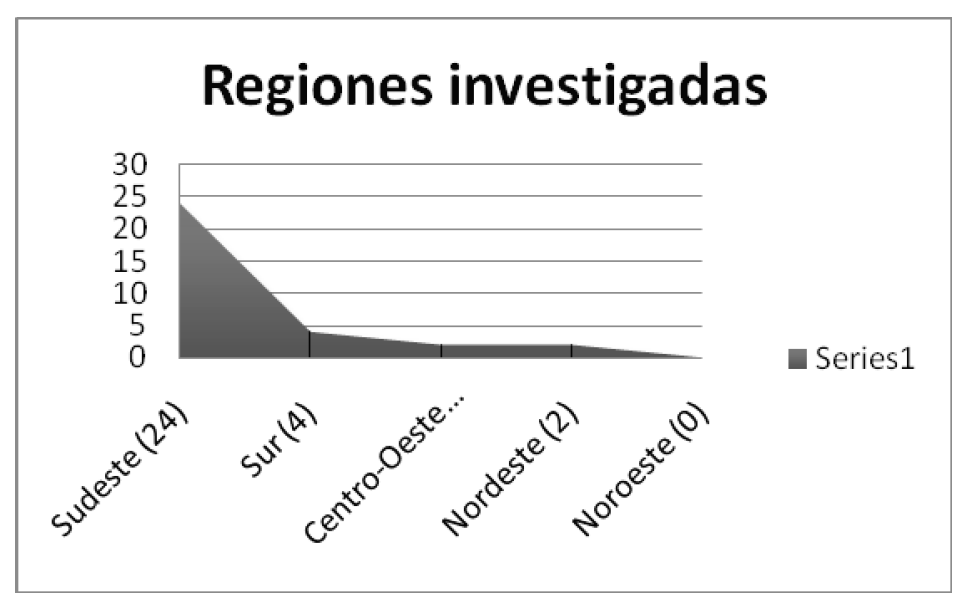

Fuente: Autora.

Para comprender el sentido de estos datos, es necesario contextualizarlos en el marco de las características socioeconómicas de cada región, para lo cual ofrecemos, en la Tabla 9, algunos indicadores relevantes. estas regiones son extremadamente heterogéneas en cuanto a sus dimensiones y población. ${ }^{3}$

Tabla 9 - Datos demográficos por región

\begin{tabular}{|c|c|c|c|c|c|c|}
\hline & Brasil & Norte & Nordeste & Centro-Oeste & Sudeste & Sur \\
\hline Área (km2) & $8.515767,049 \mathrm{~km}^{2}$ & 3.835.676,948 & 1554291,607 & 1606371 & 927.286 & 575.316 \\
\hline $\begin{array}{l}\% \text { territorio } \\
\text { nacional }\end{array}$ & $100 \%$ & $45,25 \%$ & $18,2 \%$ & $45,2 \%$ & $10,9 \%$ & $6,8 \%$ \\
\hline $\begin{array}{l}\text { \% población } \\
\text { total }\end{array}$ & $100 \%$ & 7\% (2011) & $27 \%$ (2011) & $7 \%$ (2011) & $42 \%(2011)$ & 14\% (2011) \\
\hline $\begin{array}{l}\text { Densidad } \\
\text { población }\end{array}$ & $23.8 \mathrm{hab} . / \mathrm{km}^{2}$ & 4,47 hab. $/ \mathrm{km}^{2}$ & $\begin{array}{l}\text { (2014): } \\
36,14 \text { hab./km² }\end{array}$ & 9,47 hab. $/ \mathrm{km}^{2}$ & 92,05 hab. $/ \mathrm{km}^{2}$ & $50,34 \mathrm{~h} / \mathrm{km} 2$ \\
\hline $\begin{array}{l}\text { \% población } \\
\text { rural total }\end{array}$ & $15 \%$ & 16,6 & 16,9 & 11,2 & 5,1 & 15,1 \\
\hline$\%$ PBI nacional & & $5,3 \%$ & $13,1 \%$ & $9,3 \%$ & $55,4 \%$ & $16,5 \%$ \\
\hline $\begin{array}{l}\text { Índice } \\
\text { Analfabetismo }\end{array}$ & $15,2 \%$ & $27,3 \%$ & $16,6 \%$ & $6,7 \%$ & $4,8 \%$ & $4,4 \%$ \\
\hline
\end{tabular}

Fuente: (IBGE).

Estos datos muestran que la región más industrializada, el Sudeste, atrae inversiones y población mientras que las otras regiones continúan siendo agrícolas. La región Norte, con una población predominante indígena y de inmigrantes nordestino, y el Sur con inmigrantes europeos pobres con el tiempo se volvió económicamente importante por obtener de la población el conocimiento necesario para producir. El centro-oeste, a pesar de ser una región agrícola, se construye a partir de

3. En 1967, el geógrafo Pedro Pinchas Geiger propuso una división a partir del proceso de formación del territorio brasileño, en tres grandes regiones geoeconómicas o complejos regionales (GEIGER, 1969): El Nordeste (caracterizado como la región del pasado, ya que fue allí donde tuvo lugar la colonización del país y la primera región en desarrollarse), la Amazonía (que por ser repositorio de recursos naturales del país sería la región del futuro) y el Centro-Sur (local del desarrollo presente, la región actualmente más rica y poderosa tanto económica como políticamente. Si bien ninguna división de un país en regiones es inocente, es evidente que esta clasificación estigmatiza la región del Nordeste, un área sometida a un perverso proceso histórico y que actualmente es la zona con mayores problemas económicos del país. Es interesante observar que aunque no se utilice explícitamente este criterio para dividir geopolíticamente el territorio de Brasil, los prejuicios subyacentes a esta clasificación están presentes en el imaginario cultural brasileño e impacta en las identidades de los sujetos y comunidades de cada región. 
grandes haciendas y concentración de rentas, lo cual obstaculiza la socialización de la educación. Asimismo, hay una relación entre PBI y analfabetismo: en Norte y Nordeste tienen el mayor número de personas no escolarizadas.

\subsection{Los sujetos investigados}

Por último, agrupamos las tesis relevadas según los sujetos investigados: docentes/docentes aspirantes, alumnos/exalumnos, y otros actores sociales o institucionales. Para esta clasificación seleccionamos en cada caso un único grupo de sujetos, que representaba el foco central de la investigación. Nos sorprendió encontrar en sólo una de las tesis (Alves, 2013) el centro de la investigación eran los alumnos de las escuelas de campo. Si bien otros trabajos aplicaban algún instrumento de relevamiento de datos a otros grupos, éste tenía como propósito la triangulación o la complementación de la información recogida del grupo principal de sujetos investigados. En esta etapa encontramos que 17 de las 32 tesis relevadas tenían como sujetos principales de la investigación a los docentes, 14 a otros actores y solamente 1 se centraba en los alumnos, como muestra el gráfico siguiente:

\section{Gráfico 5 - Sujetos investigados}

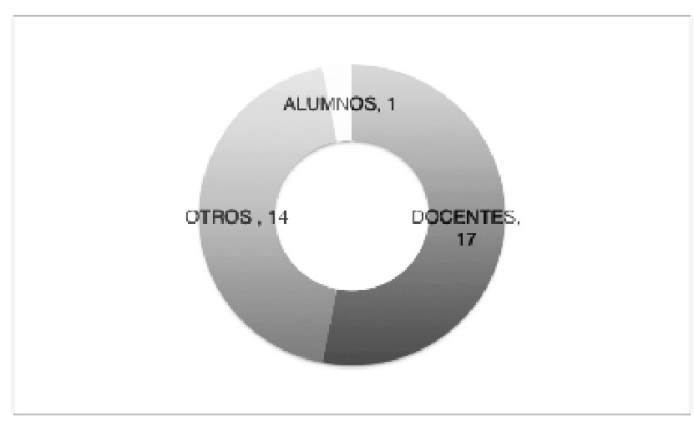

Fuente: Autora.

\section{CONCLUSIONES}

En este trabajo nos propusimos investigar qué aspectos de la educación de campo constituyen el foco de las tesis y disertaciones producidas recientemente en Brasil y publicadas en el repositorio de la BDTD, para identificar espacios para nuevas investigaciones. Un relevamiento de las tesis sobre educación de campo producidas en 2013-2014 y disponibles en la Biblioteca Digital Brasileira de Teses e Dissertações (BDTD) arrojó como resultado 32 tesis que cumplían nuestros requisitos de inclusión y exclusión. Una clasificación temática de estos trabajos muestra que hay un interés semejante en cuestiones de pedagogía y de políticas de educación de campo, si bien el tema más estudiado considerando tanto la categoría "Pedagogía" por sí misma como la totalidad de los trabajos relevados es la formación docente; este interés se agudiza cuando se consideran los sujetos investigados, con 17 de las 32 tesis relevadas dedicadas a investigar este grupo. En cuanto a la clasificación regional, la mayor parte del trabajo de campo (24 tesis) se ha realizado en el Sudeste de Brasil.

Como contrapartida a este interés en la figura de los docentes, encontramos que los alumnos de las escuelas de campo no han recibido una atención comparable a otros actores institucionales. Dado que son los estudiantes los beneficiarios de los programas de educación rural, creemos que es necesario que se lleven a cabo investigaciones sobre aspectos relativos a las subjetividades de estos actores para comprender en mayor profundidad los resultados desalentadores de algunos programas de educación de campo, especialmente en cuanto a la capacidad de retención de los alumnos. Creemos también que el hallazgo más interesante de este relevamiento ha sido comprobar que en el período 2013-2014 la Biblioteca Digital Brasileira de Teses e Dissertações (BDTD) no 
registra ninguna tesis que aborde la educación de campo en la región Centro-Oeste de Brasil, a pesar de que los indicadores de las agencias gubernamentales son profundamente preocupantes, y requieren además de políticas adecuadas un interés sostenido por parte de la academia que contribuya a mejorar y fortalecer tales políticas.

\section{ÍNDICE DE FUENTES}

\section{SECUNDARIAS}

\section{Bibliografía}

ALVES, M. (2013): Ser alguem na vida. Condição juvenil e projetos de vida de jovens moradores de um município rural da microrregião de Governador Valadares Tesis de Maestría, Universidade Federal de Minas Gerais.

AQUINO, L. (2013): Representações sociais de educandas e educandos do curso de Licenciatura em Educação do Campo sobre a leitura de texto acadêmicos. Tesis de doctorado, Universidade Federal de Minas Gerais.

ARROYO, M.; CALDART, Roseli Salete; MOLINA, Mônica Castagna (2008): Por uma educação do campo. 3. ed. Petrópolis, RJ: Vozes.

BARRADAS, C. (2013): EDUCAÇÃO DO CAMPO: formação continuada de professores do programa escola ativa em Buriti (MA). Tesis de Maestría, Universidade de Taubatçe.

BASSO, J. (2013): As escolas no campo e as salas multisseriadas no estado de São Paulo: um estudo sobre as condições da educação escolar. Tesis de Maestría, Universidade Federal de Sao Carlos.

BRASIL (2000): Evolução da Divisão Territorial do Brasil 1872-2010. Disponible enhttp://www.ibge. gov.br/home/geociencias/geografia/default_evolucao.shtm, consultado 26/1/2015

BRASIL (2008): Diretrizes operacionais para a educação básica nas escolas do campo. Brasilia:

::110:: MINISTÉRIO DA EDUCAÇÃO / Secretaria de Educação Continuada, 2004.

BRASIL (2007): Panorama da Educação do Campo. Brasilia: MEC, disponible en http://www.red-ler. org/panorama-educacao-campo.pdf

BRASIL (2010): Constituições brasileiras de 1824, 1891, 1930, 1934, 1937, 1946, 1967 e 1988. Disponible en www.camara.gov.br. Acceso 20/11/2010.

COOPER, H. and L. V. HEDGES (2009): "Research synthesis as a scientific process." The handbook of research synthesis and meta-analysis London: Sage.

COOPER, H. M. (1985): "A Taxonomy of Literature Reviews." Paper presented at the Annual Meeting of the American Educational Research Association (69th, Chicago, IL, March 31-April 4, 1985) under the title "The Literature Review: Knowledge Synthesis Activities in Education and Psychology."

DE AGUILAR, A. (2013): Escola do campo em discussão: estudo sobre os problemas de infraestrutura e políticas públicas para escolas que atendem os sujeitos rurais no município de São Carlos. Tesis de Maestría, Universidade Federal de São Carlos.

DE CAMPOS, M. (2014): Escola do campo: desafios e possibilidades para o ensino da agroecologia e educação ambiental em Araras (SP). Tesis de Maestría, Universidade Federal de São Carlos.

FEROLLA, L. (2013): Processos colaborativos na gestão pública: estudo as relações estabelecidas no contexto do Programa Nacional de Educação do Campo. São Paulo, 2013.

FERREIRA, L. (2014): Professores da zona rural em início de carreira: narrativas de si e desenvolvimento profissional. Tesis de Doctorado, Universidade Federal de São Carlos.

FREITAS, K. (2013): Formação de educadores de jovens e adultos do campo. Produções do período de 2006 à 2011. Tesis de Maestría, Universidade Federal de Viçosa.

FRIGOTTO, G.(2010): Educação e a crise do capitalismo real. São Paulo: Cortez.

GEIGER, P. P. (1969): Regionalização. Revista Brasileira de Geografia, Rio de Janeiro, 31(1), 525.

GIMENES, C. (2014): Resgate histórico da escola rurais em São João da Boa Vista - SP. Tesis de Maestría, Universidade Federal de São Carlos.

JESUS, A. (2013): Nos caminhos da escola do campo: processo de implantação da educação do campo no município de Matão, São Paulo.Tesis de Doctorado, Universidade Federal de São Carlos. 
KUHN, A. (2013): Educação do campo e ensino médio em agroecologia: estudo de caso da escola 25 de maio, Friburgo SC. Tesis de Maestría, Pontificia Universidade Católica de São Paulo.

LARCHERT, J. (2013): Resistências e seus processos educativos na comunidade negra rural quilombola do Fojo - Ba. Tesis de Doctorado, Universidade Federal de São Carlos.

LAVOURA, T. (2013): Cultura corporal e tempo livre em áreas de reforma agrária: notas a cerca da educação escolar e emancipação humana. Tesis de Doctorado, Universidade Federal de Minas Gerais.

LOPES, W. (2013): Profissionalidade docente na educação do campo. Tesis de Doctorado, Universidade Federal de São Carlos

LOPES, F. (2013): Pedagogia da terra: interfaces entre a formaçao docente, a educaçao do campo e a educaçao ambiental. Tesis de Doctorado, Universidade Federal de Uberlândia

MACHADO, V. M., \& NETA, M. D. A. P. B. (2013): Mapeamento de pesquisas sobre a juventude camponesa no movimento da educação do campo= mapping of researches about peasant youth in them ovement of education in rural areas. CAMINE: Caminhos da Educação= Camine: Ways of Education, 5(2), 43-56.

MEIRA, I. (2013): Relação entre escolarização pública e o contexto da escolado campo: Um estudo de caso no município de Boa Vista|PB. Tesis de Maestría, Universidade Federal de Paraiba.

MENEZES, L. (2013): Representações sociais sobre a educação do campo constituídas por educandos do curso de licenciatura em educação do campo. Tesis de Maestría. Universidade Federal de Minas Gerais

MOREIRA, A. (2013): Educação do Campo e docencia no cantexto da agricultura familiar: o programa escola ativa no município de Salinas MG. Tesis de Doctorado. Universidade Federal de São Carlos.

OLIVEIRA, A. (2014): Formação e trabalho no movimento sem terra (MST): processo de resistência do campesino. Tesis de Maestría, Universidade Federal de Minas Gerais.

PAULA, A. (2013): Educação do campo: desafios para a implantação de uma política educacional das escolas do campo. Tesis de Maestría, Universidade Estadual de Ponta Grossa.

PEREIRA, P. (2014): Educação rural na Paraíba. Tesis de Maestría, Universidade Federal de Paraiba.

PEREIRA, A. (2013): Territorialidade da educação do campo na região Sudoeste do Paraná na última década (2000 à 2010). Tesis de Maestría, Universidade Estadual do Oeste do Parana.

PINTO, T. (2013): A apropriação do discurso científico sobre evolução biológica por futuros professores de ciências em formação no curso de licenciatura em educação do campo da UFMG. Tesis de Maestría, Universidade Federal de Minas Gerais.

PRIEBE, M. (2013): Política pública de educação do campo: um estudo sobre a participação popular em Miradouro - Minas Gerais. Tesis de Maestría, Universidade Federal de Minas Gerais.

RANDOLPH, J. J. (2009): "A guide to writing the dissertation literature review." Practical Assessment, Research \& Evaluation 14 (13): 2.

SANTI, L. (2013): Memória autobiográficas de uma professora atuante em uma escola rural: sentidos e significados à leitura. Tesis de Maestría, Universidade Federal de Santa María.

SANTOS, S. (2013): Letramento e identidades em educação do campo. Paraná, 2013.

SAVIANI, D. (1986): Escola e Democracia. Col. Polêmicas do Nosso Tempo, São Paulo: Ed. Cortez e Ed. Autores Associados. v. 5. 10. ed., 96 p.

SILVA, N. (2013): Cultura corporal e tempo livre em áreas de reforma agrária: notas a cerca da educação escolar e emancipação humana. Tesis de Maestría, Universidade de São Paulo.

SPERANDIO, W. (2013): Formação continuada de professores na escola do campo: com a palavra os docentes do ensino médio. Tesis de Maestría, Universidade Federal do Espírito Santo.

TONDIN, C. (2013): Políticas públicas de educação para os/as agricultores/as familiares: um diálogo entre a Fetraf-Sul/CUT e o estado. Tesis de Doctorado, Pontificia Universidade Católica do Rio Grande do Sul.

WEISS, A. (2013): História de vida pessoal e profissional de uma professora do campo. Tesis de Doctorado, Universidade Federal do Espírito Santo

Recibido el 27 de agosto de 2014 Aceptado el 24 de noviembre de 2014 